\title{
Multifunctional and Multimodal Plasmonic Nanoparticles in Imaging and Therapy
}

\author{
K.V. Sokolov \\ * Department of Imaging Physics, The UT M.D. Anderson Cancer Center, Houston, TX 77030 \\ ** Department of Biomedical Engineering, University of Texas at Austin, Austin TX
}

Highly sensitive and specific molecular imaging is a key challenge in modern biology and medicine. Recently, plasmonic nanoparticles have been used to develop new assays with unprecedented sensitivity in analytical biochemistry[1-3]. This work is providing a foundation for development of new groundbreaking approaches for molecular specific imaging in live cells[4-7]. Plasmonic nanoparticles offer a number of important advantages over traditional fluorescent dyes including greatly improved photo-stability, bright signal and simple tunability of optical properties. Nanoparticles provide high surface area which can be easily modified using a variety of probe and delivery functionalities. Synthesis of hybrid core/shell nanoparticles also allows development of multimodal (e.g., MRI and optical) imaging strategies. Therefore, plasmonic nanoparticles can provide a common platform for multiple applications.

Gold nanoparticles exhibit inherent "smart" properties which are closely associated with their non-linear scattering behavior in closely spaced assemblies. We demonstrate that EGFR labeling with gold bioconjugates in cancer cells results in a more than $100 \mathrm{~nm}$ color shift and a quadratic increase in total scattering cross-section[8]. These changes give contrast ratios in excess of 10 times in images of normal and pre-cancerous epithelium in vivo. This contrast can be further increased by the use of hybrid nanoparticles with a superparamagnetic core surrounded by a gold layer[9]. The iron oxide core provides a magnetically susceptible component which can be exploited to periodically actuate cells using an external magnetic field. We demonstrated that opto-magnetic hybrid nanoparticles can significantly increase the molecular-specific contrast in optical imaging of cancer cells over imaging with gold particles alone.

As many biomolecules are located inside the cell, access to the target must involve a delivery mechanism. Therefore, novel approaches are required to tailor nanomaterials for intracellular imaging in live cells. We developed the first intracellular molecular imaging platform using gold nanoparticles which incorporate four functionalities on the same particle - targeting, endosomal uptake, cytosolic release, and improved biocompatibility[10]. Each functional element of these nanosensors is essential in providing strong molecular specific optical signal inside living cells. The utility of this contrast agent was demonstrated in the intracellular imaging of actin during cellular motility[7]. The actin mediated assembly of nanoparticles results in a red shift in scattering maxima due to plasmon resonance coupling between particles. Our experiments showed that multifunctional gold nanoparticles have a great potential as a complementary contrast agent to quantum dots and other probes for the optical imaging of molecular intracellular processes.

References

[1] R. Elghanian et al., Science (Washington, D. C.), 227 (1997) 1078.

[2] J. J. Storhoff et al., Nature Biotechnology, 22 (2004) 883.

[3] C. Soennichsen et al., Nature Biotechnology, 23 (2005) 741.

[4] J. Yguerabide and E. E. Yguerabide, Journal of Cellular Biochemistry, (2001) 71.

[5] K. Sokolov et al., Cancer Research, 63 (2003)1999. 
[6] J. Aaron et al., Nano Letters, 9 (2009) 3612.

[7] S. Kumar et al., Nano Letters, 7 (2007) 1338.

[8] J. Aaron et al., Journal of Biomedical Optics, 12 (2007).

[9] J. S. Aaron et al., Optics Express, 14 (2006) 12930.

[10] S. Kumar, J. Aaron, and K. Sokolov, Nat Protoc, 3 (2008) 314.

[11] This research was supported by NIH grants CA103830, EB008101, CA135315 and CA135315-02S1. 